\title{
Bloqueo auriculoventricular congénito y embarazo ¿Qué hacer con el marcapaso?
}

\section{Congenital atrioventricular block and pregnancy What to do with the pacemaker?}

\author{
Jerson Quitián, Guillermo Mora • Bogotá, D.C. (Colombia)
}

\section{Resumen}

El bloqueo aurículoventricular congénito (BAVC) es una lesión del nodo AV que produce alteración de la transmisión de los impulsos auriculares a los ventrículos y que puede manifestarse clínicamente antes o después del nacimiento. Es raro encontrar esta patología en mujeres embarazadas, sin embargo esto puede variar debido a que en la actualidad se corrigen defectos cardiacos de manera quirúrgica más frecuentemente y se implantan marcapasos de manera más precoz. Presentamos un caso de una mujer con BAVC y embarazo, se discute el manejo del marcapaso en este grupo de pacientes. (Acta Med Colomb 2012; 37: 152-157)

Palabras clave: bloqueo auriculoventricular congénito, marcapaso.

\begin{abstract}
The congenital atrioventricular block (CAVB) is an AV node lesion that produces alteration in the transmission of atrial impulses to the ventricles that may be manifested clinically before or after birth. This condition is rare in pregnant women; however, this can vary because nowadays heart defects are corrected surgically more frequently and pacemakers are implanted earlier. We present a case of a pregnant woman with CAVB, and discuss pacemaker management in this group of patients. (Acta Med Colomb 2012; 37: 152-157)
\end{abstract}

Keywords: congenital atrioventricular block, pacemaker.
Dr. Jerson Quitián Moreno: Fellow Cardiología Universidad El Bosque. Servicio de Electrofisiología Fundación Santa Fe de Bogotá; Dr. Guillermo Mora Pabón: Servicio de Electrofisiología Fundación Santa Fe de Bogotá; Profesor Asociado Departamento de Medicina Interna Universidad Nacional de Colombia. Bogotá, D.C. (Colombia).

Correspondencia. Dr. Guillermo Mora Pabón. Bogotá, D.C. (Colombia).

E-mail: gmorap@unal.edu.co

Recibido: 15/XII/2011 Aceptado: 01/VIII/2012

\section{Introducción}

El bloqueo auriculoventricular congénito (BAVC) es una lesión del nodo AV que produce alteración de la transmisión de los impulsos auriculares a los ventrículos, puede manifestarse clínicamente antes del nacimiento o en la mayoría de los casos después de él. Aparece en forma aislada o familiar, presentando una incidencia variable entre 0.12 - 1/2.500 neonatos vivos, dependiendo de la metodología de los estudios publicados (1). Presentamos un caso de una paciente embarazada con bloqueo AV congénito sintomático y discutimos las diversas posibilidades de manejo en este grupo de pacientes con respecto a la necesidad y uso del marcapaso.

\section{Caso clínico}

Una paciente de 28 años de edad con embarazo de 11 semanas, consultó por cuadro clínico de un día de evolución consistente en episodios de palpitaciones asociado a disnea en reposo. Refiere haber presentado cuatro episodios sincopales, el primero hace ocho años y el último hace dos meses. Niega otra sintomatología. No hay antecedentes de importancia personales ni familiares, no hay enfermedades autoinmunes en la madre.

El examen físico de ingreso fue normal excepto por la auscultación de ruidos cardiacos bradicárdicos. En el trazo electrocardiográfico realizado se documenta bloqueo AV completo con QRS angosto (Figura 1) y en estudio Holter bloqueo AV de primer grado seguido de bloqueo auriculoventricular completo intermitente (Figura 2). Los estudios solicitados incluyendo función tiroidea, función renal, electrolitos, perfil autoinmune y ecocardiograma transtorácico fueron normales. Se considera que dado lo sintomático de su bloqueo AV se debe implantar un marcapaso definitivo.

Es valorada por el servicio de ginecoobstetricia quienes dan la indicación de, si las condiciones hemodinámicas de la paciente lo permiten, se debe implantar el marcapaso hasta la semana 16 para permitir un adecuado desarrollo embrionario. Una vez cumplida la ventana de tiempo sugerida se 


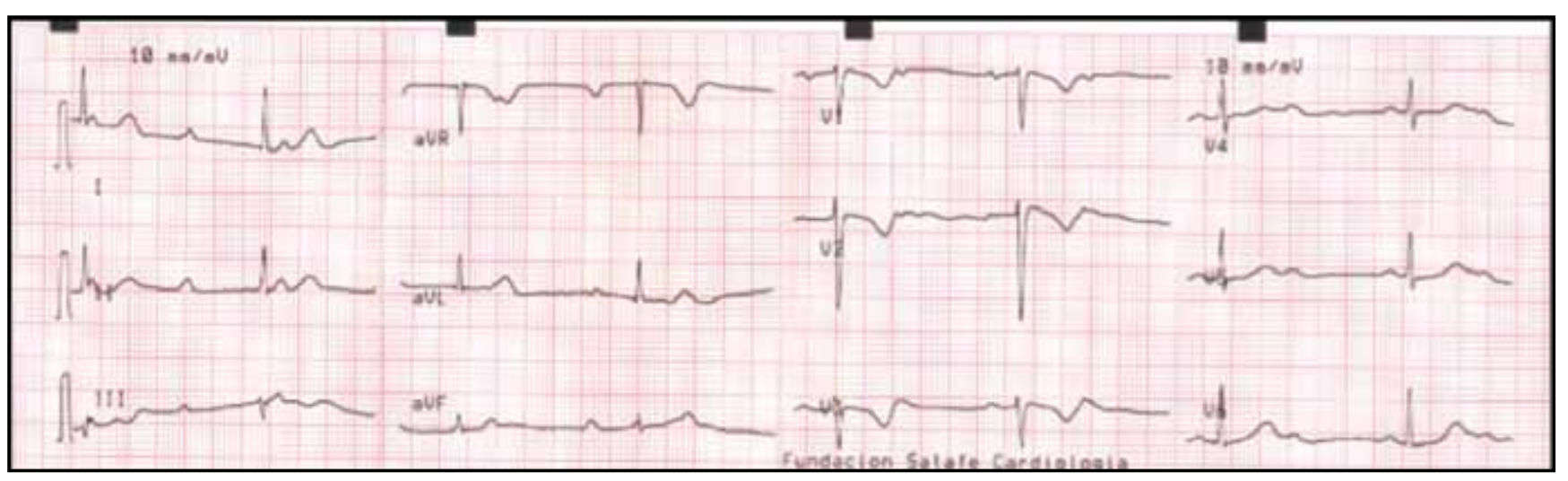

Figura 1. Electrocardiograma que muestra bloqueo AV completo con QRS angosto. Nótese que hay regularidad del RR y el PP y aunque inicialmente podría considerarse como un Bloqueo AV 2:1 los PR varían lo que confirma el diagnóstico de bloqueo AV completo.

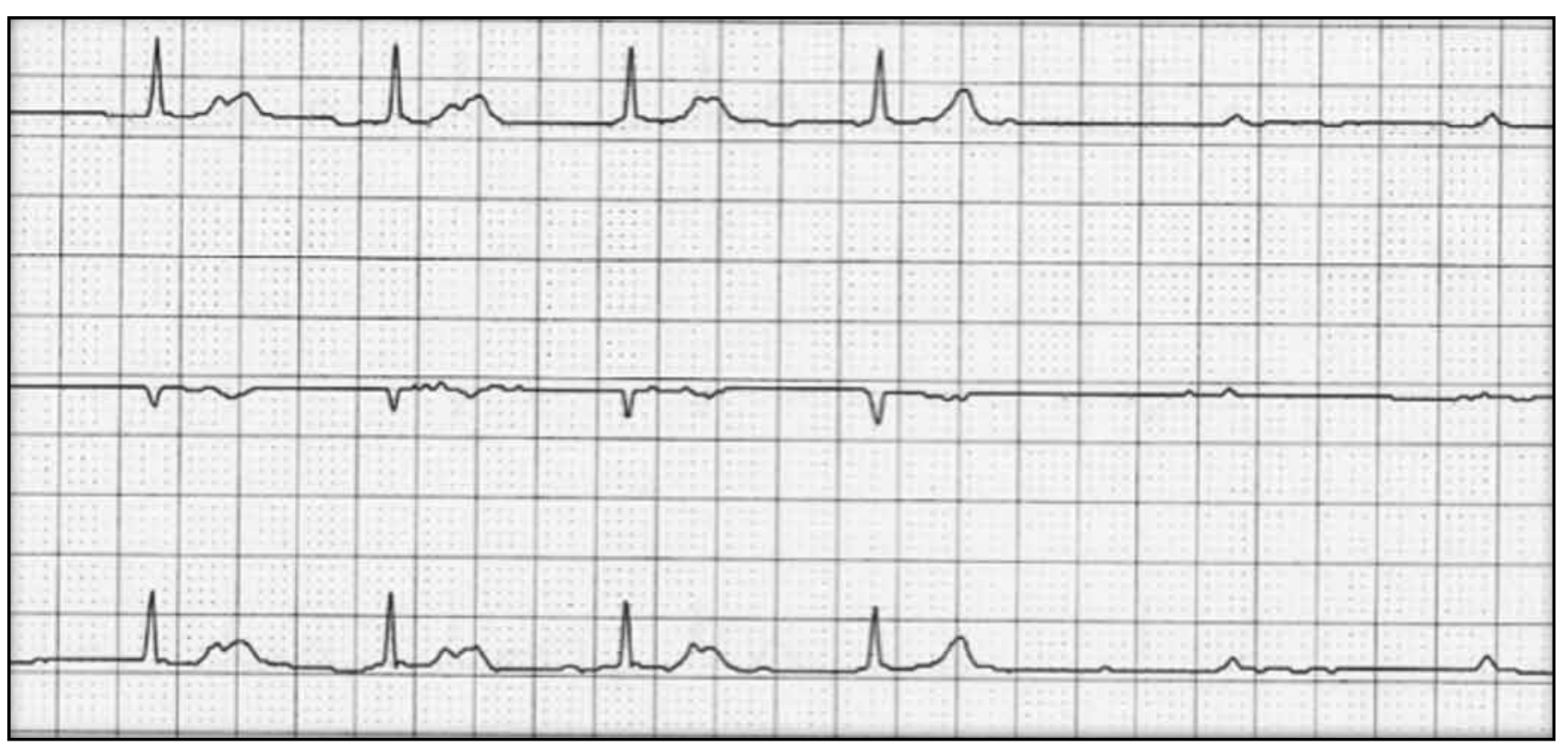

Figura 2. Monitoría de Holter que muestra bloqueo AV de primer grado seguido de Bloqueo auriculoventricular completo.

realiza el implante de marcapaso bicameral (ACCENT de St Jude Medical) por vía subclavia izquierda, sin complicaciones, con adecuados parámetros de estimulación y sensado. La dosis de radiación fue de $19 \mathrm{mGy}$. La paciente es llevada en la semana 36 a cesárea por parto distósico y tiene un recién nacido sano con adecuada talla y peso.

\section{Discusión}

\section{El bloqueo AV congénito}

Aunque no hay una definición totalmente aceptada de bloqueo AV congénito se puede considerar como los trastornos de la conducción AV que se desarrollan desde la etapa fetal y que se manifiestan al nacimiento o posteriormente sin que exista una causa externa que lo explique (por ejemplo drogas, cirugía cardiaca o endocarditis). La primera descripción clínica corresponde a Morquio en 1901 y la primera confirmación electrocardiográfica fue presentada por Van den Heuvel en 1908 (1, 2).

El bloqueo AV congénito se acompaña frecuentemente de la coexistencia de cardiopatía estructural y, en casos de anatomía cardiaca normal, es frecuente la asociación con enfermedades autoinmunes clínicas o subclínicas, como el lupus eritematoso sistémico y/o a la presencia de autoanticuerpos (AC) anti-Ro y anti-La, en las madres de los hijos afectados $(3,4)$.

Los factores de riesgo incluyen el diagnóstico previo de BAVC en algún miembro de la familia, títulos elevados de AC anti-Ro+ (títulos superiores a 1:16), la presencia de AC anti-Ro+ (SS-A) acompañados de AC anti-La+ (SS-B) y ciertos haplotipos HLA (HLA DR3) en la madre (5-6).

Se ha descrito una evidente relación entre la presencia de AC SS-A/Ro o SS-B/La maternos y la aparición de BAVC 
fetal. Así, $90 \%$ de las madres de hijos afectados tienen antiRo+ y $80 \%$ tienen anti-La+ (7). Estos anticuerpos maternos son tipo IgG que atraviesan la placenta y penetran en la circulación fetal, uniéndose al Ag La y Ro cardiacos, afectando el miocardio y el tejido de conducción produciendo inflamación y posterior fibrosis $(4,7)$.

El tiempo de la positivización de los AC y la aparición de criterios clínicos de enfermedad conectiva materna puede ocurrir años después del nacimiento de un feto afectado. Se ha propuesto la hipótesis que una infección viral (coxsackie, citomegalovirus) puede ser el factor desencadenante de la expresión en la superficie de membrana de los Ag Ro y $\mathrm{La}$, los cuales son proteínas intracelulares de localización intracitoplasmática e intranuclear respectivamente $(8,9)$. En estos casos, de bloqueo AV congénito, el nivel de bloqueo es suprahisiano, lo que explica que el QRS sea angosto y que muchos pacientes cursen inicialmente de forma asintomática por un ritmo nodal que puede acelerarse con el ejercicio. Nuestra paciente tiene un QRS estrecho que sugiere un compromiso suprahisiano.

La segunda causa de BAVC es el desarrollo embrionario anormal del nodo AV y ramas proximales del haz de His, asociado frecuentemente a anomalías cardiacas estructurales. Se conoce que en un $25-50 \%$ de casos se acompaña de una cardiopatía estructural, siendo la más frecuente la transposición de grandes arterias (TGA) o los síndromes poliesplénicos (10). Aunque estrictamente, el bloqueo surgido como consecuencia de una cirugía no es congénito éste ocurre en aproximadamente $1.7-3 \%$ de los procedimientos asociados a corrección de cardiopatías congénitas $(11,12)$ y se puede manifestar clínicamente en la edad adulta en mujeres embarazadas.

Las indicaciones para el manejo con marcapaso de pacientes con BAVC no son claramente establecidas. Un estudio prospectivo demostró que la implantación de marcapaso producía mejoría en la supervivencia, en el número de eventos sincopales, en la disfunción miocárdica y la regurgitación mitral, incluso en pacientes asintomáticos (13).

Sigue siendo raro el hecho de encontrar esta patología en mujeres embarazadas; sin embargo, esto puede variar debido a que en la actualidad se corrigen quirúrgicamente defectos cardiacos de forma más frecuente, siendo esto un riesgo para el desarrollo de BAV como complicación posoperatoria. De esta manera nos veremos comúnmente enfrentados a la mujer embarazada con bloqueo AV o con marcapaso implantado por esta patología.

\section{Implicaciones para el embarazo del BAVC}

La gestación tiene un efecto arritmogénico, el cual puede incluir desde sensación de palpitaciones, arritmias supraventriculares, hasta taquicardia ventricular en presencia $o$ ausencia de enfermedad cardiaca. Las palpitaciones pueden ser desencadenadas por los cambios del embarazo en cuanto al aumento de la frecuencia cardiaca, disminución de la resistencia periférica y aumento de volumen (14).
En un embarazo normal el gasto cardiaco comienza a aumentar a partir de las cinco semanas y continúa hasta la mitad del embarazo, cuando es $45 \%$ más alto en comparación con las mujeres no embarazadas (15). El aumento del volumen sistólico se produce un poco más tarde a las ocho semanas y alcanza un máximo hacia las 20 semanas. Este aumento es esencial para satisfacer los mayores requerimientos hemodinámicos del embarazo y depende de la frecuencia cardiaca y del volumen sistólico. A partir de la semana cinco la frecuencia cardiaca se eleva hasta la semana 32 alcanzando un $25 \%$ más de la frecuencia basal. Después de la mitad del embarazo, el gasto cardiaco permanece estable sin cambios significativos hasta el momento del parto donde las contracciones uterinas dan una carga adicional al sistema cardiovascular al desplazar sangre a la circulación central.

Dado que la mayoría de pacientes BAVC no puede lograr un aumento suficiente de la frecuencia cardíaca, el gasto cardiaco durante el embarazo dependerá principalmente del aumento del volumen sistólico. Es por ello que algunas mujeres embarazadas con BAVC pueden permanecer asintomáticas, pero otras pueden desarrollar síncope, disnea, o dilatación ventricular.

No está claro si en la mujer embarazada con BAVC, sin marcapaso, el estado hemodinámico se mantiene estable durante el parto y después de éste, teniendo en cuenta el aumento del trabajo cardiaco y la pérdida aguda de sangre que a menudo se produce durante este periodo. Casos aislados y pequeñas series de casos de BAVC en mujeres embarazadas han sido reportados en la literatura (16), por lo tanto no hay pautas establecidas para el tratamiento clínico de este grupo de pacientes. Se sabe también que además de enfermedad de conducción, $10-15 \%$ de la descendencia afectada tendrá riesgo de desarrollar cardiomiopatía (17).

La muerte cardiaca súbita (MCS) puede ser la manifestación clínica inicial en pacientes con BAV congénito previamente asintomáticos. Los mecanismos postulados son pausas sin ritmo de escape adecuado o arritmias ventriculares mediadas por alteración en la dispersión de la refractariedad condicionada por la bradiarritmia. Los principales factores de riesgo para el desarrollo de la MCS en este contexto son la presencia de cardiopatía congénita estructural asociada, el QT prolongado y una frecuencia cardiaca menor a 50 lpm. En estos pacientes hay indicación de implante de marcapaso definitivo. Durante los últimos años se ha obtenido información observacional, que sugiere que el implante de marcapaso podría aumentar la sobrevida de pacientes con BAVC asintomático sin estos factores, relacionado posiblemente con la disminución de arritmias ventriculares pausa dependientes $(18,19)$.

\section{Escenarios clínicos de bloqueo AV congénito y embarazo \\ - Mujer embarazada con marcapaso permanente}

Los marcapasos permanentes se utilizan con mayor frecuencia en mujeres jóvenes con bradicardia permanente, 
por ello es posible que algunas mujeres en edad reproductiva, con bradicardia severa secundaria a BAVC tendrán un marcapaso permanente implantado antes del embarazo.

Existe poca literatura acerca del tipo y programación de marcapaso que se debe usar en este grupo de pacientes; en un estudio se describen 11 pacientes embarazadas con marcapaso permanente de las cuales seis se encontraban en programación DDDR, uno en VDD, uno como DDI, uno en AAIR por síndrome de seno enfermo, un paciente con función dual de marcapaso más cardiodesfibrilador y uno con marcapaso biventricular (20). Sin embargo, no hay datos para establecer por qué cada paciente tenía este tipo de dispositivos.

No hay estudios que definan cuál es la mejor programación que se debe hacer a los marcapasos en pacientes embarazadas en trabajo de parto. El problema está resuelto en las mujeres que tiene marcapaso DDD con buena función sinusal en las cuales las necesidades metabólicas y hemodinámicas activarán el nodo sinusal y el marcapaso estimulará el ventrículo "siguiendo" la activación auricular. En las pacientes con marcapasos unicamerales ventriculares (VVI/VVIR) es posible que sea necesario reprogramar el dispositivo para dejarlo en frecuencias cardiacas mayores a las habituales (80-90 vs 50-60) para satisfacer los requerimientos hemodinámicos. Igualmente se deberá aumentar la frecuencia de base del marcapaso en pacientes con disfunción sinusal (aún con marcapaso DDD/DDDR) dado que el nodo sinusal no responderá espontáneamente a las necesidades metabólicas de las pacientes. Sin embargo, la decisión de cuál es la mejor frecuencia cardiaca se deberá individualizar en cada caso.

\section{- Mujer embarazada con bloqueo AV sin marcapaso permanente}

Aunque el riesgo de muerte súbita es impredecible en pacientes asintomáticos, algunos expertos recomiendan que la implantación del marcapaso se deba retrasar el mayor tiempo posible, debido al riesgo de infección y la necesidad de cambios periódicos del dispositivo. Sin embargo, como se expuso previamente, otros autores han recomendado el implante de marcapasos una vez se diagnostique el bloqueo. Las guías colombianas de electrofisiología de la Sociedad Colombiana de Cardiología consideran que es razonable el implante de marcapaso permanente en pacientes con cardiopatía congénita y compromiso hemodinámico debido a bradicardia sinusal o pérdida de la sincronía auriculoventricular. (Recomendación IIA (nivel de evidencia C) (21).

Algunas mujeres asintomáticas sin marcapasos que se embarazan pueden finalmente convertirse en sintomáticas (mareo, disnea, síncope) o incluso desarrollar insuficiencia cardiaca durante embarazo como consecuencia de la incapacidad de aumentar el gasto cardiaco debido a la imposibilidad de aumentar la frecuencia cardiaca (22). Se ha reportado que los embarazos en mujeres con BAVC sin marcapaso se pueden asociar con alta mortalidad materna y fetal $(23,24)$.
En las pacientes sintomáticas en el primer o segundo trimestre, generalmente se recomienda la implantación de marcapasos permanente; por otra parte después de la mitad del embarazo es raro el desarrollo de síntomas, incluso en pacientes sin marcapasos teniendo en cuenta que el gasto cardiaco o la frecuencia cardiaca no cambian significativamente en esta fase del embarazo $(22,25)$.

Si presenta síntomas en este periodo (final del embarazo), se recomienda marcapaso temporal seguido por inducción del parto a la mayor brevedad posible. Después del parto, algunos de los cambios hemodinámicos que se producen durante el embarazo, incluyendo aumento del volumen sanguíneo, hemodilución y la resistencia vascular periférica, rápidamente vuelven a los niveles antes del embarazo. Por lo tanto, las pacientes deben ser reevaluadas en el posparto y si los síntomas persisten, entonces se debe discutir la implantación de un marcapaso definitivo.

La complicación más frecuente del implante de un marcapaso permanente en mujeres embarazadas es la irritación de la piel y ulceración en el área de implantación que se ha relacionado con el crecimiento mamario $(16,26,27)$. Otras complicaciones son dolor y extrasístoles (28).

La realización de imágenes en las mujeres embarazadas presenta un desafío único a causa de la preocupación por el riesgo de radiación del embrión. Cuando es necesario el estudio radiográfico o fluoroscópico, se debe tener en cuenta que si el útero está fuera del campo de trabajo, el embrión está expuesto a radiación dispersa y la dosis es mínima.

Mayores valores de dosis se producen cuando el útero se encuentra dentro del campo de trabajo. En este caso, la dosis de radiación a un embrión a partir de un examen radiográfico o radioscópico depende del espesor de la pared abdominal de la paciente, la dirección de la proyección (anteroposterior, posteroanterior o lateral) y qué tan profundo se encuentra el útero (según la edad gestacional). Los efectos potenciales incluyen muerte prenatal, retraso del crecimiento intrauterino, retraso en el tamaño de la cabeza, retraso mental grave, cociente de inteligencia reducido, malformaciones y cáncer infantil (29). Estos efectos dependen de la dosis de radiación del embrión y la etapa de desarrollo en que la exposición se produce (30).

Comúnmente se utiliza un protector de plomo para proteger al útero de la radiación externa difusa (dispersión que emanan de los tejidos expuestos) si el área de interés se encuentra fuera del útero. Sin embargo, debido a que la dosis de la radiación dispersa es mínima, el uso de blindaje de plomo se deja al criterio del médico, teniendo en cuenta que ofrece a la paciente una sensación de protección y seguridad (31).

En 1977 la comisión nacional de protección de medidas contra la radiación emitió una declaración en la que se refería al riesgo de anormalidad en el feto, considerando insignificante si la exposición era $50 \mathrm{mGy}$ o menos. El riesgo aumenta significativamente en dosis superiores a $150 \mathrm{mGy}$ (31). Por lo tanto, la exposición del feto a la radiación en los 
procedimientos de implante de marcapaso sería muy baja y la posibilidad de incremento de anomalías en el feto o la pérdida del embarazo muy rara (31). En nuestra paciente la exposición fue de sólo $19 \mathrm{mGy}$, con lo que el riesgo para el feto fue mínimo y al momento del parto no se detectaron anomalías.

\section{- Manejo del parto}

En lo posible se prefiere que las mujeres embarazadas con BAVC, ingresen al hospital un par de días antes de la fecha prevista de parto para anticipar la necesidad de soporte hemodinámico.

Recientemente se ha sugerido que una importante opción de tratamiento para las pacientes sin marcapaso puede ser el uso de marcapaso temporal durante la progresión del trabajo de parto; varios informes de casos han documentado buenos resultados con este tratamiento, aunque no es claro si la estimulación temporal es realmente necesaria y exista justificación absoluta para su uso $(32,33)$. Otros autores sugieren que las mujeres con BAVC, que no requieren un marcapaso permanente (asintomáticas, sin factores de riesgo), pueden ser manejados durante el parto sin el soporte temporal, eliminando así los riesgos asociados con este procedimiento (34).

\section{Conclusiones y recomendaciones}

No es fácil el abordaje de estos casos ni existen guías de recomendación específica por lo que algunos pacientes necesitarán un manejo individualizado; sin embargo, cuando hay síntomas, evidencia del diagnóstico eléctrico de bloqueo AV y embarazo, se debe contar con conceptos claros con la evidencia que se tiene actualmente, sustentado a su vez con el apoyo multidisciplinario del cardiólogo, electrofisiólogo y obstetra.

En la Tabla 1 se presentan las recomendaciones que proponemos de manejo de pacientes con embarazo y bloqueo AV con o sin presencia de marcapaso.

\section{Conflictos de interés}

No se declara conflictos de interés.

Tabla 1. Recomendaciones para pacientes embarazadas con marcapaso o posible necesidad de él.

\footnotetext{
Embarazo más marcapaso permanente

* MCP DDD/AAI = con buena función del nodo sinusal, no hacer cambios. **MCP DDD/DDD/AAI = mala función sinusal, aumentar FC 80-90 lpm ***MCP VVI = Aumentar FC 80-90 lpm.

Embarazo sin MCP *sintomática

$1^{\circ}$ y $2^{\circ}$ trimestre: Implante de marcapaso definitivo.

Tercer trimestre: implante de marcapaso transitorio e inducción del parto. Revaloración posparto.
}

MCP: Marcapaso, DDD: Marcapaso bicameral, AAI: Marcapaso unicameral localizado en la aurícula, FC Frecuencia cardiaca, lpm: latidos por minuto, VVI: Marcapaso unicameral localizado en el ventrículo.

\section{Referencias}

1. Michaelsson M, Engle MA. Congenital complete heart block: an international study of the natural history. Cardiovasc Clin 1972; 4: 85-101.

2. Plant RK, Steven RA. Complete A-V block in a fetus. Am Heart J 1945; 30: 615-8.

3. Ramsey-Goldamn R, Hom D, Deng J. Anti SS-A antibodies and fetal outcome in maternal systemic lupus erythematosus. Arthritis Rheum 1986; 29: 1.269-1.273.

4. Olah KS, Gee H. Fetal heart block associated with maternal ani-Ro (SS-A) antibody. Current management. A review. Br J Obstet Gynaecol 1991; 98: 751-755.

5. Scheib JS, Waxman J. Congenital heart block in successive pregnancies: a case report and evaluation of risk with therapeutic consideration. Obstet Gynecol 1989; 73: $481-484$.

6. Taylor PV, Taylor KF, Norman A, Griffiths S, Scott JS. Prevalence of maternal Ro (SS-A) and LA (SS-B) autoantibodies in relation to congenital heart block. $\mathrm{Br}$ J Rheumatol 1988; 27: 128-132.

7. Chua S, Ostman-Smith I, Sellers S, Redman CWG. Congenital heart block with hydropsfetalis treated with high-dose dexamethasone; a case report. Eur J Obstet Gynecol Reprod Biol 1991; 42: 155-158.

8. Chameides L, Truex RC, Vetter V, Rashkind WJ, Galioto FM, Noonan JA Association of maternal systemic lupus erythematosus with congenital complete heart block. N Engl J Med 1977; 297: 1.204-1.207.

9. Taylor PV, Scott JS, Gerlis LM, Path FR, Esscher E, Scott O. Maternal antibodies against fetal cardiac antigens in congenital complete heart block. $N$ Engl J Med 1986; 315: 667-672.

10. Schmidt KG, Ulmer HE, Silverman NH. Perinatal outcome of fetal complete atrioventricular block: a multicenter experience. J Am Coll Cardiol 1991; 17: $1.360-1.365$

11. Weindling SN, Saul JP, Gamble WJ, Mayer JE, Wessel D, Walsh EP. Duration of complete atrioventricular block after congenital heart disease surgery. Am J Cardiol 1998; 82: 525-527.

12. Yildirim SV, Tokel K, Saygili B, Varan B. The incidence and risk factors of arrhythmias in the early period after cardiac surgery in pediatric patients. Turk $J$ Pediatr 2008; 50: 549-553.

13. Richard A. Friedman, MD. Congenital AV Block Pace Me Now or Pace Me Later? Circulation 1995; 92: 283-285.

14. Shotan A, Ostrzega E, Mehra A, Johnson JV, Elkayam U. Incidence of arrhythmias in normal pregnancy and relation to palpitations, dizziness, and syncope. Am J Cardiol 1997; 79: 1061-4

15. Duvekot JJ, Peeters LL. Maternal cardiovascular hemodynamic adaptation to pregnancy. Obstet Gynecol Surv 1994; 49: S1-S14.

16. Ginns HM, Hollinrake K. Complete heart block in pregnancy treated with an internal cardiac pacemaker. J Obstet Gynaecology Br Commonw 1970; 77: 710-712.

17. Deborah M. Friedman, Carolina Llanos, Peter M. Izmirly, Brigit Brock, John Byron. Evaluation of Fetuses in the Preventive IVIG Therapy for Congenital Heart Block (PITCH) study. Arthritis Rheum 2010; 62(4): 1138-1146.

18. Esscher E, Michaëlsson M. Q-T interval in congenital complete heart block. Pediatr Cardiol 1983; 4: 121-4.

19. HidakaN, Chiba Y, Fukushima K, Wake N. Pregnant Women with Complete Atrioventricular Block: Perinatal Risks and Review of Management. PACE 2011; 34: $1161-1176$

20. Thaman R, Curtis S, Faganello G, Szantho GV. Cardiac outcome of pregnancy in women with a pacemaker and women with untreated atrioventricular conduction block. Europace 2011; 13: 859-863.

21. Sociedad Colombiana de Cardiología y Cirugía Cardiovascular, Colegio Colombiano de Electrofisiología Cardiovascular. Guías colombianas de electrofisiología cardiovascular 2011; 18 Supl 3: 225-227.

22. Lau CP, Lee CP, Wong CK, Cheng CH, Leung WH. Rate responsive pacing with a minute ventilation sensing pacemaker during pregnancy and delivery. Pacing Clin Electrophysiol 1990; 13: 158-163.

23. Epstein JR, Altman HE. Heart block in pregnancy: report of two cases. Med Ann Dist Columbia 1951; 20: 660-663.

24. Mendelson CL. Disorders of the heart beat during pregnancy. Am J Obstet Gynecol 1956; 72: 1268-1301.

25. Gudal M, Kervancioglu C, Oral D, Gurel T, Erol C, Sonel A. Permanent pacemaker implantation in a pregnant woman with the guidance of ECG and twodimensional echocardiography. Pacing Clin Electrophysiol 1987; 10: 543-545.

26. Middleton EB, Lee YC. Pregnancy associated with cardiac pacemaker generator implanted in the abdominal wall. Obstet Gynecol 1971; 38: 272-275.

27. Andersen C, Oxho H, Arnsbo P, Lybecker H. Pregnancy and cesarean section in a patient with a rate-responsive pacemaker. Pacing Clin Electrophysiol 1989; 12: $386-391$ 
28. Jaffe R, Gruber A, Fejgin M, Altaras M, Ben-Aderet N. Pregnancy with an artificial pacemaker. Obstet Gynecol Surv 1987; 42: 137-139.

29. Wagner LK, Lester RG, Saldana LR. Exposure of the pregnant patient to diagnostic radiations: a guide to medical management. Madison, Wis: Medical Physics Publishing, 1997.

30. Wagner LK, Hayman LA. Pregnancy and women radiologists. Radiology 1982; 145: 559-562.

31. Cynthia H, McCollough, PhD, Beth A. Schueler, PhD. Radiation exposure and pregnancy: When should we be concerned? Radiographics 2007; 27: 909-918.

32. Dalvi BV, Chandhuri A, Kulkarni HL, Kale PA. Therapeutic guidelines for congenital complete heart block. Obstet Gynecol 1992; 79: 802-804.

33. Mehta S, Goswami D, Temple A. Successful pregnancy outcome in a patient with complete heart block. J Postgrad Med 2003; 49: 98.

34. Hidaka N, Chiba Y, Kurita T, Satoh S, Nakano H. Is intrapartum temporary pacing required for women with complete atrioventricular block?. An analysis of seven cases. BJOG 2006; 113: 605-607. 